\title{
Long non-coding RNAs in nucleus pulposus cell function and intervertebral disc degeneration
}

\author{
Zheng $\mathrm{Li}^{1}$ | Xingye $\mathrm{Li}^{2}$ | Chong Chen ${ }^{1}$ | Shugang $\mathrm{Li}^{1}$ | Jianxiong Shen ${ }^{1}$ | \\ Gary Tse $^{3,4}$ | Matthew T. V. Chan ${ }^{4}$ | William K. K. Wu $u^{4,5}$
}

\author{
${ }^{1}$ Department of Orthopaedic \\ Surgery, Peking Union Medical College \\ Hospital, Chinese Academy of Medical \\ Sciences and Peking Union Medical College, \\ Beijing, China \\ ${ }^{2}$ Department of Orthopedic Surgery, Beijing \\ Jishuitan Hospital, Fourth Clinical College \\ of Peking University, Jishuitan Orthopaedic \\ College of Tsinghua University, Beijing, \\ China \\ ${ }^{3}$ Department of Medicine and \\ Therapeutics, The Chinese University of \\ Hong Kong, Hong Kong, China \\ ${ }^{4} \mathrm{Li}$ Ka Shing Institute of Health Sciences, The \\ Chinese University of Hong Kong, Hong \\ Kong, China \\ ${ }^{5}$ Department of Anaesthesia and Intensive \\ Care and State Key Laboratory of Digestive \\ Diseases, The Chinese University of Hong \\ Kong, Hong Kong, China
}

\section{Correspondence}

Shugang Li and Jianxiong Shen, Department of Orthopaedic Surgery, Peking Union Medical College Hospital, Chinese Academy of Medical Sciences and Peking Union Medical College, Beijing, China.

Emails: lishugangpumc@163.com; sjxpumch@163.com

Funding information

National Natural Science Foundation of China (NSFC), Grant/Award Number: 81330044; The Capital Health Research and Development of Special, Grant/Award Number: 2016-1-4096

\begin{abstract}
Intervertebral disc degeneration (IDD) is the major cause of low back pain which incurs a significant public-health and economic burden. The aetiology of IDD is complex, with developmental, genetic, biomechanical and biochemical factors contributing to the disease development. Deregulated phenotypes of nucleus pulposus cells, including aberrant differentiation, apoptosis, proliferation and extracellular matrix deposition, are involved in the initiation and progression of IDD. Non-coding RNAs, including long non-coding RNAs (IncRNAs), have recently been identified as important regulators of gene expression. Research into their roles in IDD has been very active over the past 5 years. Our review summarizes current research regarding the roles of deregulated IncRNAs (eg, RP11-296A18.3, TUG1, HCG18) in modulating nucleus pulposus cell functions in IDD. These exciting findings suggest that specific modulation of IncRNAs or their downstream signalling pathways might be an attractive approach for developing novel therapeutics for IDD.
\end{abstract}

\section{1 | INTRODUCTION}

Low back pain is a common but debilitating disorder, creating a huge public-health and economic burden. Enormous efforts have been put forth to investigate the pathogenesis and optimise the clinical management of low back pain. ${ }^{1-4}$ The aetiology of low back pain is manifold, among which intervertebral disc degeneration (IDD) is a

Zheng Li, Xingye Li and Chong Chen are the co-first authors. major but poorly understood contributing factor. ${ }^{5-7}$ The pathogenesis of IDD can be attributed to a myriad of risk factors, including lifestyles (eg, smoking, alcohol consumption, occupation), genetic predisposition, and ageing. ${ }^{8-12}$ However, the precise cellular and molecular mechanisms linking these factors to IDD development still remain unclear. In this respect, a growing body of evidence has suggested that nucleus pulposus (NP) cells are crucial for preserving the integrity of intervertebral discs (IVD) via their roles in producing 
extracellular matrix (ECM) components, such as aggrecan and type II collagen, and secretion of cytokines. ${ }^{13-15}$ Importantly, deregulation of NP cell functions, including aberrant cell proliferation, apoptosis and ECM synthesis/degradation have all been implicated in the development of IDD. ${ }^{13,16-20}$

Long non-coding RNAs (IncRNAs) are a group of non-coding RNAs which are longer than 200 nucleotides in length. Due to their long length, IncRNAs possess unique ability to adopt a variety of complex secondary and tertiary structures, allowing them to perform specific functions, such as catalysis, metabolite sensing and precise protein recognition. ${ }^{21-24}$ The predicted secondary structure of Xist, which is the best-characterised IncRNA to date, is shown in Figure 1 as an example of structural complexity of IncRNAs. LncRNAs have no or little capacity for protein coding, but could modulate gene expression at epigenetic (eg, DNA methylation, histone modification), transcriptional (eg, recruitment of transcriptional factors) and post-transcriptional (eg, sponging of microRNAs, regulation of mRNA stability) levels. ${ }^{25-29}$ An increasing number of studies have demonstrated IncRNA deregulation in different kinds of morbidities, including neoplastic, inflammatory, infectious and orthopaedic diseases. ${ }^{30-35}$ As a key control of gene expression, IncRNAs play pivotal roles in regulating cellular phenotypes, including differentiation, apoptosis, proliferation, metabolism, migration and invasion. ${ }^{29,36-39}$ Recently, some studies suggested that IncRNAs are critical players in the development of IDD. In this review article, we summarise the current knowledge regarding the deregulation of IncRNAs in IDD in relation to their effects on NP cell proliferation, apoptosis and ECM synthesis/degradation. The potential clinical utilities of IncRNAs as therapeutic targets for the management of IDD are also discussed.

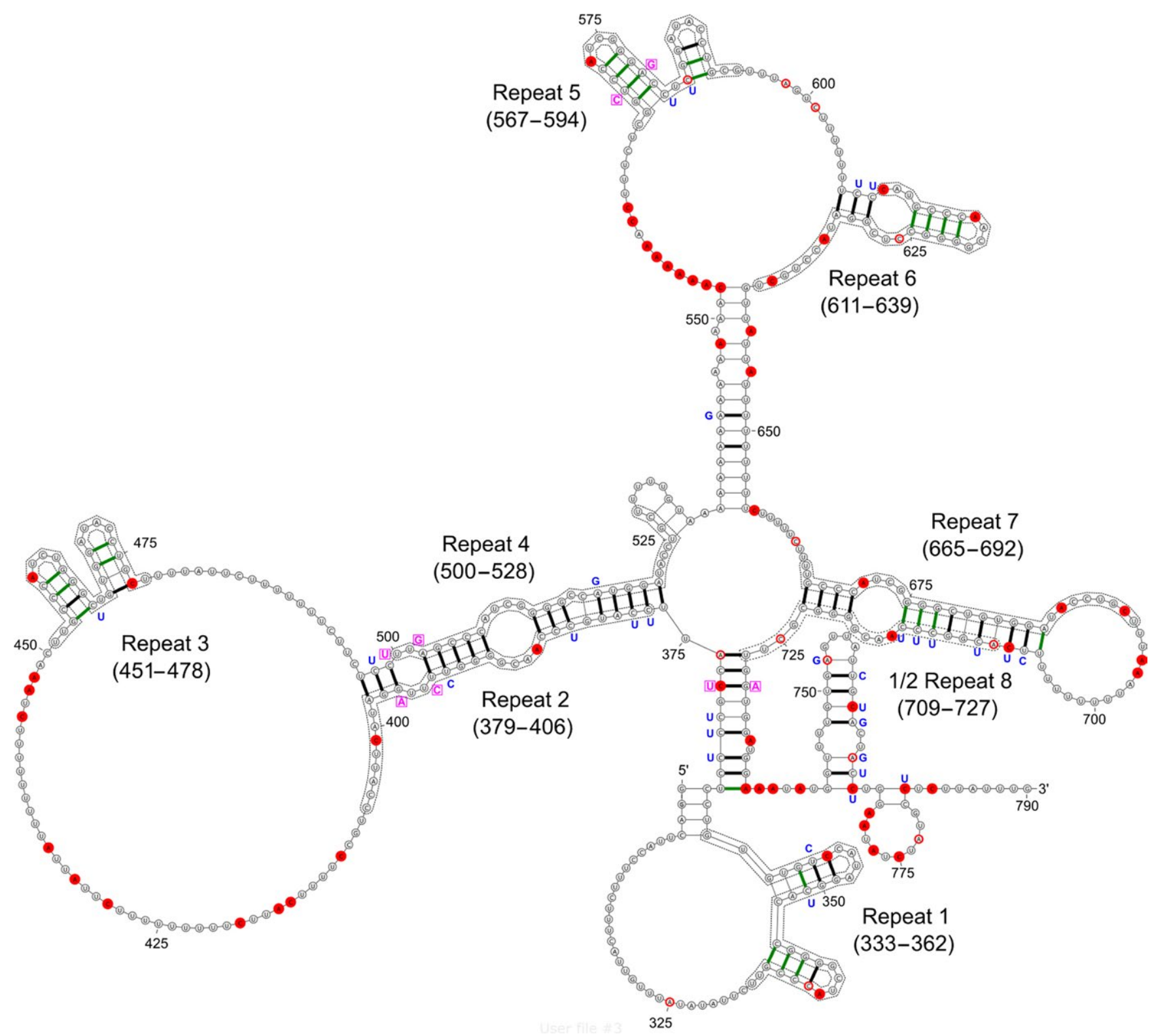

FIG URE 1 Predicted secondary structure of Xist, the best-characterised IncRNA to date (adapted from Wikimedia Commons) 


\section{2 | LnCRNA EXPRESSION PROFILING IN INTERVERTEBRAL DISC DEGENERATION}

Genome-wide IncRNA profiling by microarray or RNA sequencing followed by validation of candidate IncRNAs with reverse transcription-quantitative PCR (RT-qPCR) is a common approach to identify and confirm differential IncRNA expression in a specific disease state. ${ }^{40-42}$ The samples analysed often include clinical specimens and cultured cells. ${ }^{43,44}$

Wan and colleagues ${ }^{45}$ used the IncRNA-mRNA microarray to profile differentially expressed IncRNAs and mRNAs in NP cells isolated from degenerative and non-degenerative IVD samples. A total of 116 IncRNAs (67 upregulated and 49 downregulated) and 260 mRNAs were found to be significantly differentially expressed with fold-change larger than ten. KEGG (Kyoto Encyclopedia of Genes and Genomes) pathway and IncRNA-mRNA co-expression network analyses showed that the 10 most significantly deregulated IncRNAs were involved in several known degenerative alterations, such as chondrocyte differentiation, collagen fibril organisation and proteoglycan metabolism. In particular, by RTqPCR validation, the authors found that Fas-associated protein factor-1, which potentiates the Fas-induced apoptosis, and its nearby enhancer-like IncRNA RP11-296A18.3 were both upregulated in the degenerative IVDs, implicating the involvement of RP11-296A18.3 in aberrant NP cell apoptosis. The same team ${ }^{46}$ subsequently conducted an integrative analysis on datasets from mRNA, miRNA, circular RNA and IncRNA microarrays and further depicted the comprehensive landscape of RNAs in human IDD by illustrating the interactions among members from different RNA classes during the disease development. Chen and colleagues ${ }^{47}$ also re-analysed the datasets from these two studies. A total of 135 upregulated and 170 downregulated IncRNAs together with 2133 upregulated and 1098 downregulated mRNAs were identified, among which seven IncRNAs (LINC00917, CTC-523E23.5, CTD-2246P4.1, RP11-363G2.4, RP4-639J15.1, RP11-38F22.1 and $A C 005082.12)$ were predicted to play key functional roles by IncRNA-mRNA co-expression network analysis, in particular regulation of cell migration by interactions of CTD-2246P4.1 and LINC00917 with SPHK1.
Unbiased genome-wide transcriptome analysis by highthroughput sequencing has depicted the RNA landscapes of different human tissues at unprecedented resolutions. Zhao and colleagues ${ }^{48}$ performed transcriptome sequencing on an Illumina platform to identify deregulated IncRNAs in central NP tissues isolated from IDD patients as compared with those from control patients. A total of 1854 IncRNAs were found to be differentially expressed with fold-change more than two, in which 1530 IncRNAs were predicted to target 6386 genes through cis-regulatory effects. KEGG pathway analysis for these target genes revealed that IncRNAs were involved in regulation of focal adhesion, lysosome function and mitogen-activated protein kinase (MAPK) signalling. Bioinformatic analysis of interactions among IncRNAs, microRNAs, and mRNAs suggested that the upregulated IncRNA PART1 might mediate the pathogenic effect via sponging miR-34a and miR-148a to derepress their corresponding target genes E2F3, VEGFA and ACVR1 in IDD.

The abovementioned studies have unequivocally demonstrated that IncRNA expression is significantly deregulated in degenerative IVD tissues and NP cells and strongly suggested that IncRNAs might be functionally involved in IDD pathogenesis (Table 1). Nevertheless, it is noteworthy that different studies have given rise to different results. For instance, the top 10 upregulated and downregulated IncRNAs identified in the RNA sequencing study by Zhao and colleagues ${ }^{48}$ showed no overlap with the top deregulated IncRNAs $(P<.05$; fold-change $>10)$ identified in the microarray study by Wan and colleagues. ${ }^{45}$ Whether the discrepancy arose as a result of the use of different clinical tissues or profiling platforms, however, is unclear.

\section{3 | FUNCTIONAL ROLES OF LNCRNAS IN INTERVERTEBRAL DISC DEGENERATION}

\section{$3.1 \mid$ RP11-296A18.3}

Aberrant proliferation of NP cells is implicated in IDD pathogenesis. ${ }^{19}$ Wang and colleagues ${ }^{49}$ determined the functional role of RP11-296A18.3, an aberrantly upregulated IncRNA, in the development of IDD. The authors demonstrated that knockdown of RP11296A18.3 suppressed the human NP cell proliferation and reduced

TAB LE 1 Long non-coding RNA expression profiles in intervertebral disc degeneration

\begin{tabular}{|c|c|c|c|c|c|c|}
\hline No. & Method & Sample & Filtering criteria & Upregulated & Downregulated & Reference \\
\hline 1 & $\begin{array}{l}\text { Microarray (GSE56081) } \\
\text { RT-PCR }\end{array}$ & $\begin{array}{l}\text { Degenerate } \\
\text { disc tissues }\end{array}$ & $P<.05 ;$ Fold-change $>10$ & 67 IncRNAs & 49 IncRNAs & 45 \\
\hline 2 & $\begin{array}{l}\text { Integrative analysis of } \\
\text { mRNA, miRNA, circular } \\
\text { RNA and IncRNA } \\
\text { (GSE56081) microarrays }\end{array}$ & $\begin{array}{l}\text { Degenerate } \\
\text { disc tissues }\end{array}$ & $P<.05 ;$ Fold-change $>2$ & 2234 IncRNAs & 847 IncRNAs & 46 \\
\hline 3 & $\begin{array}{l}\text { Bioinformatics re-analysis } \\
\text { of dataset GSE56081 }\end{array}$ & $\begin{array}{l}\text { Degenerate } \\
\text { disc tissues }\end{array}$ & $P<.05 ;$ Fold-change $>1.5$ & 135 IncRNAs & 170 IncRNAs & 47 \\
\hline 4 & RNA sequencing & $\begin{array}{l}\text { Degenerate } \\
\text { disc tissues }\end{array}$ & Fold-change $>2$ & 916 IncRNAs & 938 IncRNAs & 48 \\
\hline
\end{tabular}


the expression of collagen I and matrix metalloproteinase (MMP)-13. Target prediction, RT-qPCR and luciferase assays further revealed that RP11-296A18.3 acted as a competing endogenous RNA for miR-138 and thereby derepressing its target HIF1A. In addition, the interrelationship among RP11-296A18.3, miR-138 and HIF1A was confirmed in human IDD tissues by immunochemistry and RT-qPCR. These data indicated that RP11-296A18.3 promoted human NP cell proliferation and modulated ECM deposition via regulating the miR138-HIF1A axis. These results also suggested that this IncRNA might be a therapeutic target in IDD.

\section{2 | TUG1}

Over-activation of Wnt/ $\beta$-catenin signalling is casually linked to IDD development through multiple mechanisms, including induction of cellular senescence and abnormal ECM deposition/degradation. ${ }^{50}$ The taurine upregulated gene 1 (TUG1) is a newly identified IncRNA frequently upregulated in human malignancies ${ }^{30}$ and mechanistically linked to $\mathrm{Wnt} / \beta$-catenin activation. ${ }^{51}$ Chen and colleagues ${ }^{52}$ investigated the expression of IncRNA TUG1 in degenerative NP tissues and its functional role of in IDD in relation to $\mathrm{Wnt} / \beta$-catenin signalling. The authors first collected 30 NP samples from patients with lumbar disc herniation and 18 NP tissues from control subjects with lumbar spine trauma. TUG1 expression was found to be significantly upregulated in lumbar disc herniation NP samples and positively associated with Wnt1 and $\beta$-catenin expression. In NP cells, enforced expression of TUG1 increased the expression of Wnt1 and $\beta$-catenin and enhanced tumour necrosis factor (TNF)- $\alpha$-induced cell senescence and apoptosis. Concordantly, TUG1 increased the expression of pro-apoptotic Bax and caspase- 3 but reduced the expression of anti-apoptotic $\mathrm{Bcl}-2$. In addition, overexpression of TUG1 upregulated ECM-degrading genes (ie, MMP3 and ADAMTS5) but downregulated ECM-coding genes (ie, aggrecan and COL2A1). The pro-senescence, pro-apoptotic and ECM-modulating effects of TUG1 overexpression were blocked by the Wnt/ $\beta$-catenin inhibitor XAV-939. Knockdown of TUG1 produced the opposite effects. These data suggested that aberrant upregulation of TUG1 in NP cells resulted in senescence, apoptosis and suppression of ECM synthesis through the Wnt/ $\beta$-catenin pathway. Targeting TUG1 might thus be a potential therapeutic strategy for the treatment of IDD.

\section{3 | HCG18}

$\mathrm{Xi}$ and colleagues ${ }^{53}$ demonstrated that the expression of the IncRNA HCG18 (HLA complex group 18) transcribed from chromosome 6 was significantly upregulated in NP tissues isolated from patients with herniated or bulging discs and was positively associated with the grade of disc degeneration. Mechanistically, HCG18 was found to function as an endogenous sponge for miR-146a-5p to inhibit cell proliferation, promote cell apoptosis, and enhance release of chemoattractants for macrophages in NP cells. Conversely, knockout of miR-146a-5p expression produced the opposite effects. Mechanistically, TRAF6, an upstream positive regulator of nuclear factor (NF)- $\mathrm{kB}$ signalling and a direct target gene of miR-146a-5p, and phosphorylation of NF-kB p65 at Serine 536 were found to be upregulated upon HCG18 overexpression. The positive correlation between HCG18 and TRAF6 expression was also confirmed in clinical NP tissues. To this end, knockdown of TRAF6 by small hairpin RNA reversed HCG18-induced NP proliferative arrest and apoptosis. These results suggested that aberrant overexpression of HCG18 might promote IDD development through inducing human NP cell death via derepressing the TRAF6-NFkB pathway that is normally inhibited by miR-146a-5p.

\section{4 | MALAT1}

MALAT1 (metastasis associated lung adenocarcinoma transcript 1) also known as NEAT2 is a IncRNA with physiological functions in different cellular processes, including alternative splicing, nuclear organisation and epigenetic modulation of gene expression. ${ }^{54}$ Zhang and colleagues ${ }^{55}$ first demonstrated that the expression level of MALAT1 was significantly reduced in NP cells isolated from IDD patients as compared with control. Functionally, restored expression of MALAT1 promoted caspase 3 activity (an apoptotic marker) and reduced the secretion of interleukins (IL)- 1 and -6 . These findings suggested that reduced MALAT1 expression might participate in IDD development via inducing NP cell apoptosis and secretion of pro-inflammatory cytokines.

\subsection{SNHG1}

Small nucleolar RNA host gene 1 (SNHG1) is a host to eight small nucleolar RNAs. The overexpression of SNHG1 has been demonstrated in hepatocellular carcinoma, lung cancer, oesophageal cancer and colorectal cancer and was associated with poorer prognosis or more aggressive phenotypes. The mechanisms involved in the oncogenic action of SHNG1 include sponging of microRNAs (eg, miR-101-3p, and miR-195, miR-338) and activation of Wnt/ $\beta$-catenin signalling. ${ }^{56,57}$ Recently, Tan and colleagues ${ }^{58}$ investigated the role of SNHG1 in the progression of IDD. They first demonstrated that SNHG1 expression was significantly upregulated in IDD tissues as compared with control samples. Higher expression of SNHG1 was also positively correlated with the grade of disc degeneration. Functionally, enforced expression of SNHG1 induced the NP cell proliferation and promoted the cyclin D1 and PCNA expression. Mechanistically, SNHG1 repressed miR-326 to promote NP cell proliferation. The negative correlation between SNHG1 and miR-326 was also demonstrated in clinical IDD samples. These results suggested that the IncRNA SNHG1 played a crucal role in IDD development through inducing aberrant NP cell proliferation.

\section{6 | H19}

$\mathrm{H} 19$ is an imprinted oncofoetal IncRNAs with high expression levels during embryogenesis but is nearly undetectable in most adult tissues. ${ }^{59}$ Wang and colleagues ${ }^{60}$ demonstrated that the expression of $\mathrm{H} 19$ was upregulated in clinical IDD specimens and in cultured 
TAB LE 2 Functional characterisation of the IncRNAs in IDD

\begin{tabular}{lllll}
\hline LncRNAs & Expression & Functional role & & Related genes \\
\hline RP11-296A18.3 & Up & Proliferation matrix metalloproteinase & miR-138 HIF1A & Reference \\
\hline TUG1 & Up & Senescence apoptosis ECM-degrading & Wnt $\beta$-catenin & 52 \\
\hline HCG18 & Up & Proliferation apoptosis & miR-146a-5p TRAF6 \\
\hline MALAT1 & Down & Apoptosis inflammatory cytokines & & 55 \\
SNHG1 & Up & Proliferation & miR-326 & 58 \\
\hline H19 & Up & Senescence ECM-degrading & miR-22 LEF1 & 60 \\
NEAT1 & Up & ECM-degrading & ERK/MAPK & ADAMTS5 \\
\hline linc-ADAMTS5 & Down & Aggrecan degradation & 64 \\
\hline
\end{tabular}

NP cells undergoing oxidative stress-induced cellular senescence. Functionally, enforced expression of $\mathrm{H} 19$ increased oxidative stressinduced degeneration through inducing NP cell senescence, enhancing MMP and ADAMTS-5 protein expression as well as inhibiting the NP cell proliferation via activating $\mathrm{Wnt} / \beta$-catenin signalling. To this end, $\mathrm{H} 19$ directly sponged miR-22 via interacting with the 3 '-untranslated region to alleviate its inhibition on LEF1, which is a transcription factor required for $\beta$-catenin-driven transcription of Wnt target genes.

\subsection{NEAT1}

NEAT1 (nuclear paraspeckle assembly transcript 1) is a novel IncRNA localised specifically to nuclear paraspeckles and its overexpression has been documented in many human malignancies. ${ }^{61}$ Ruan and colleagues ${ }^{62}$ assessed the functional role and the associated mechanism of NEAT1 in IDD development. The authors first demonstrated that that the expression levels of NEAT1, p21 and p53 were all upregulated in the degenerative NP tissues as compared with the control NP samples. In this regard, enforced expression of NEAT1 upregulated the ECM-degrading ADAMTS5 and MMP13 expression and suppressed aggrecan and collagen II expression through the MAPK/extracellular signal-regulated kinase (ERK) signalling. These findings suggested that the IncRNA NEAT1 might contribute to IDD development by tipping the balance between ECM deposition and degradation by NP cells in an MAPK/ ERK-dependent manner.

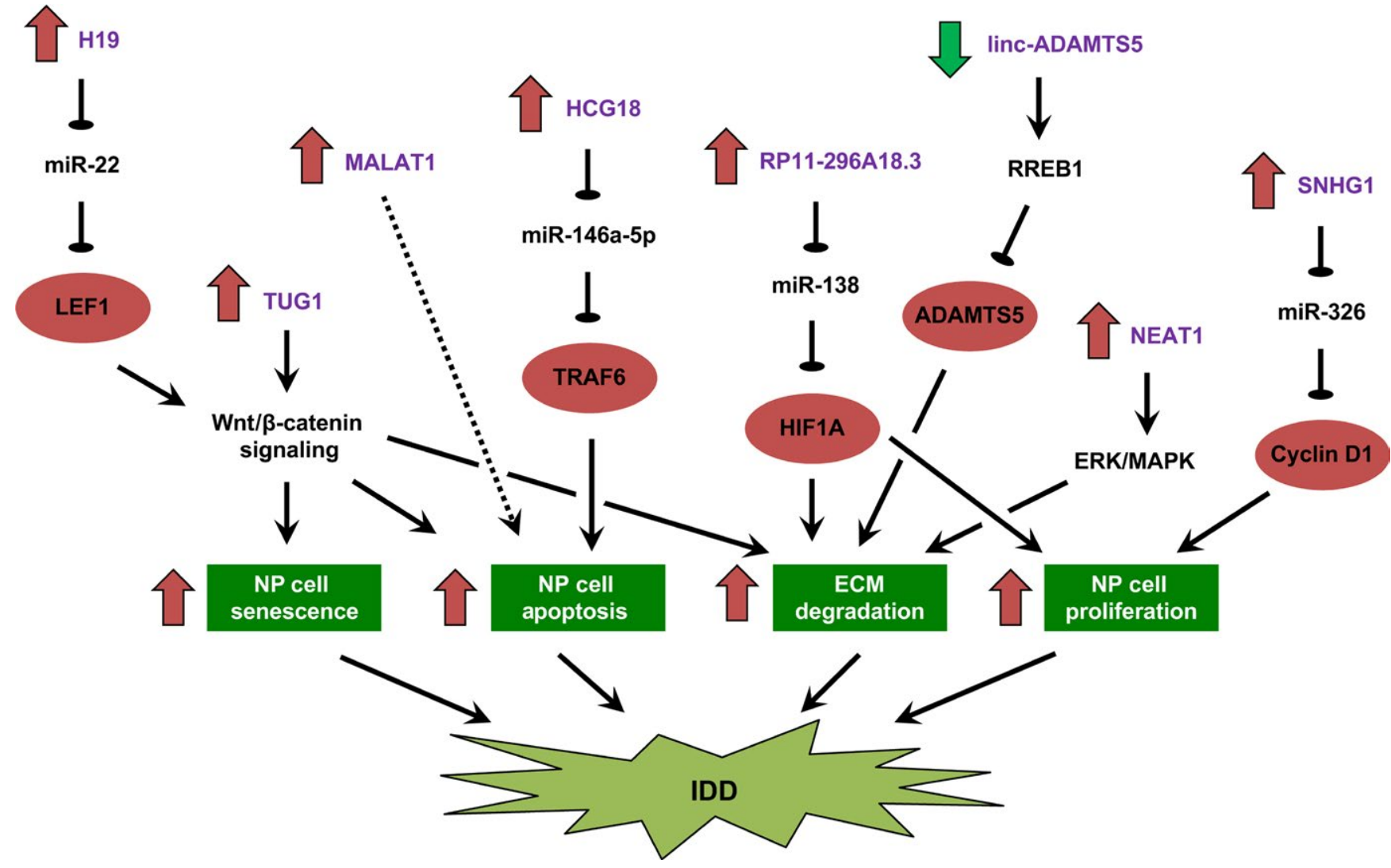

FIGURE 2 Deregulation of intracellular signalling and functions of nucleus pulposus cells by IncRNAs in intervertebral disc degeneration 


\section{8 | linc-ADAMTS5}

Inflammation has been linked to increased aggrecan degradation in IDD. ${ }^{63}$ Wang and colleagues ${ }^{64}$ investigated the functional role of linc-ADAMTS5, which is a IncRNA transcribed in opposite direction to the ADAMTS5 (a gene encoding an aggrecan-degrading enzyme), in IDD. The authors found that the expression of linc-ADAMTS5 was progressively downregulated with advancing grades of degeneration in human IDD. Bioinformatic analysis, RNA immunoprecipitation, in vitro binding assay and functional studies showed that linc-ADAMTS5 physically interacted with the splicing factor proline/glutamine-rich (SFPQ) to facilitate the recruitment of RREB1 (a transcription factor) to the binding site within ADAMTS5 promoter to promote chromatin remodelling and thus epigenetic silencing. Concordantly, lincADAMTS5 and RREB1 expression were negatively correlated with ADAMTS5 expression in clinical NP samples. These data suggested that progressive downregulation of linc-ADAMTS5 could promote aggrecan degradation in IDD development via derepressing the expression of ADAMTS5 (Table 2).

\section{4 | CONCLUSION}

Intervertebral disc degeneration is a common pathogenic process underlying low back pain. However, its molecular and cellular pathogenesis remains largely unknown. Emerging evidence supported that IncRNAs play critical roles in orthopaedic diseases. In particular, an increasing number of studies have hinted at the functional roles of IncRNAs in IDD and have shed new light on their clinical use as potential therapeutic targets. Through IncRNA profiling and RT-PCR, several key deregulated IncRNAs in both NP cells and tissues have been identified. However, functional studies of these IncRNAs still remain limited. LncRNAs might be involved in IDD initiation and progression through modulating NP cell proliferation, apoptosis and ECM deposition (Figure 2). Deregulated IncRNAs might also alter cytokines release (eg, IL-1, IL-6) or modulate response to cytokines (eg, TNF- $\alpha$-induced cell senescence and apoptosis) in NP cells and thereby contributing to inflammation. Treatment for IDD might thus be achieved through targeting deregulated IncRNAs, for example, using IncRNA-specific small interfering RNA delivered by nanoparticles or lipid-encapsulation as well as small-molecule inhibitors that perturb that interaction of particular IncRNA with its RNA or protein partners. Apart from treatment, early detection and prognostication of IDD remain clinically challenging. It is noteworthy that certain circulating or tissue IncRNAs have been proposed as novel diagnostic and prognostic markers in human cancers. Nevertheless, systematic identification and validation of IncRNAs as biomarkers in IDD are still lacking. More translational works are thus needed to maximise the clinical potentials of IncRNAs in IDD.

Over the recent years, a rapid expansion of technologies has greatly accelerated the discovery and functional characterisation of disease-associated IncRNAs. These technological advancements with respect to IncRNA profiling interactome analysis (comprehensively reviewed by Jathar et $\mathrm{al}^{65}$ ) have promoted the identification and mechanistic study of IncRNAs in other diseases. It is anticipated these methods could be applied to the study of IDD-associated IncRNAs and shed new light on the molecular pathogenesis of IDD.

\section{ACKNOWLEDGEMENTS}

This work was supported by grants from the National Natural Science Foundation of China (NSFC) (Grant Numbers: 81330044) and The Capital Health Research and Development of Special (Grant Numbers: 2016-1-4096).

\section{REFERENCES}

1. Becker A, Held H, Redaelli M, et al. Implementation of a guideline for low back pain management in primary care - a cost-effectiveness analysis. Spine. 2012;37:701-710.

2. Samartzis D, Karppinen J, Mok F, Fong DY, Luk KD, Cheung KM. A population-based study of juvenile disc degeneration and its association with overweight and obesity, low back pain, and diminished functional status. J Bone Joint Surg Am. 2011;93:662-670.

3. Tajerian M, Alvarado S, Millecamps $M$, et al. DNA methylation of SPARC and chronic low back pain. Mol Pain. 2011;7:65.

4. Speed C. Low back pain. BMJ. 2004;328:1119-1121.

5. Arana E, Kovacs FM, Royuela A, et al. Modic changes and associated features in Southern European chronic low back pain patients. Spine J. 2011;11:402-411.

6. Tegeder I, Lotsch J. Current evidence for a modulation of low back pain by human genetic variants. J Cell Mol Med. 2009;13(8B):1605-1619.

7. Omair A, Holden M, Lie BA, Reikeras O, Brox Jl. Treatment outcome of chronic low back pain and radiographic lumbar disc degeneration are associated with inflammatory and matrix degrading gene variants: a prospective genetic association study. BMC Musculoskelet Disord. 2013;14:105.

8. Loreto C, Musumeci G, Castorina A, Martinez G. Degenerative disc disease of herniated intervertebral discs is associated with extracellular matrix remodeling, vimentin-positive cells and cell death. Ann Anat. 2011;193:156-162.

9. Phillips KL, Jordan-Mahy N, Nicklin MJ, Le Maitre CL. Interleukin-1 receptor antagonist deficient mice provide insights into pathogenesis of human intervertebral disc degeneration. Ann Rheum Dis. 2013;72:1860-1867.

10. Roberts S, Evans H, Trivedi J, Menage J. Histology and pathology of the human intervertebral disc. J Bone Joint Surg Am. 2006;88(Suppl 2):10-14.

11. Inoue N, Espinoza Orias AA. Biomechanics of intervertebral disk degeneration. Orthop Clin North Am. 2011;42:487-499.

12. Adams MA, Roughley PJ. What is intervertebral disc degeneration, and what causes it? Spine. 2006;31:2151-2161.

13. Li Z, Yu X, Shen J, Chan MT, Wu WK. MicroRNA in intervertebral disc degeneration. Cell Prolif. 2015;48:278-283.

14. Wang SZ, Rui YF, Lu J, Wang C. Cell and molecular biology of intervertebral disc degeneration: current understanding and implications for potential therapeutic strategies. Cell Prolif. 2014;47:381-390.

15. Gruber HE, Ingram JA, Leslie K, Hanley EN Jr. Cellular, but not matrix, immunolocalization of SPARC in the human intervertebral disc: decreasing localization with aging and disc degeneration. Spine. 2004;29:2223-2228.

16. Li HR, Cui Q, Dong ZY, Zhang JH, Li HQ, Zhao L. Downregulation of miR-27b is involved in loss of type II collagen by directly targeting 
matrix metalloproteinase 13 (MMP13) in human intervertebral disc degeneration. Spine. 2016;41:E116-E123.

17. Li Z, Shen J, Wu WK, et al. Leptin induces cyclin D1 expression and proliferation of human nucleus pulposus cells via JAK/STAT, PI3K/ Akt and MEK/ERK pathways. PLoS ONE. 2012;7:e53176.

18. Li Z, Yu X, Liang J, Wu WK, Yu J, Shen J. Leptin downregulates aggrecan through the p38-ADAMST pathway in human nucleus pulposus cells. PLoS ONE. 2014;9:e109595.

19. Yu X, Li Z, Shen J, et al. MicroRNA-10b promotes nucleus pulposus cell proliferation through RhoC-Akt pathway by targeting HOXD10 in intervetebral disc degeneration. PLoS ONE. 2013;8:e83080.

20. Li Z, Shen J, Wu WK, et al. The role of leptin on the organization and expression of cytoskeleton elements in nucleus pulposus cells. J Orthop Res. 2013;31:847-857.

21. Yu X, Li Z. Long non-coding RNA HOTAIR: a novel oncogene (Review). Mol Med Rep. 2015;12:5611-5618.

22. Yu X, Li Z. Long non-coding RNA growth arrest-specific transcript 5 in tumor biology. Oncol Lett. 2015;10:1953-1958.

23. Li Z, Yu X, Shen JX. Long non-coding RNAs: emerging players in osteosarcoma. Tumor Biol. 2016;37:2811-2816.

24. Huang CS, Liu SG, Wang HJ, Zhang ZC, Yang Q, Gao F. LncRNA PVT1 overexpression is a poor prognostic biomarker and regulates migration and invasion in small cell lung cancer. Am J Transl Res. 2016;8:5025-5034.

25. Li P, Xue WJ, Feng Y, Mao QS. Long non-coding RNA CASC2 suppresses the proliferation of gastric cancer cells by regulating the MAPK signaling pathway. Am J Transl Res. 2016;8:3522-3529.

26. Liu CB, Lin JJ. Long noncoding RNA ZEB1-AS1 acts as an oncogene in osteosarcoma by epigenetically activating ZEB1. Am J Transl Res. 2016;8:4095-4105.

27. Ma GX, Tang MQ, Wu YQ, Xu XM, Pan F, Xu RA. LncRNAs and miRNAs: potential biomarkers and therapeutic targets for prostate cancer. Am J Transl Res. 2016;8:5141-5150.

28. Li J, Li Z, Zheng W, et al. LncRNA-ATB: an indispensable cancerrelated long noncoding RNA. Cell Prolif. 2017;50(6). https://doi. org/10.1111/cpr.12381.

29. Li J, Lian Y, Yan C, et al. Long non-coding RNA FOXP4-AS1 is an unfavourable prognostic factor and regulates proliferation and apoptosis in colorectal cancer. Cell Prolif. 2017;50(1). https://doi.org/10.1111/ cpr.12312.

30. Li Z, Shen J, Chan M, Wu W. TUG1: a pivotal oncogenic long noncoding RNA of human cancers. Cell Prolif. 2016;49:471-475.

31. Ma X, Qi S, Duan Z, et al. Long non-coding RNA LOC554202 modulates chordoma cell proliferation and invasion by recruiting EZH2 and regulating miR-31 expression. Cell Prolif. 2017;50(6). https:// doi.org/10.1111/cpr.12388.

32. Zhao J, Gao Z, Zhang C, Wu H, Gu R, Jiang R. Long non-coding RNA ASBEL promotes osteosarcoma cell proliferation, migration and invasion by regulating microRNA-21. J Cell Biochem. 2018; https://doi. org/10.1002/jcb.26671. [Epub ahead of print].

33. Zhang $X$, Tang $X, M a ~ L$, et al. Schisandrin B down-regulated IncRNA BCYRN1 expression of airway smooth muscle cells by improving miR150 expression to inhibit the proliferation and migration of ASMC in asthmatic rats. Cell Prolif. 2017;50(6). https://doi.org/10.1111/ cpr.12382.

34. Wei B, Wei W, Zhao B, Guo X, Liu S. Long non-coding RNA HOTAIR inhibits miR-17-5p to regulate osteogenic differentiation and proliferation in non-traumatic osteonecrosis of femoral head. PLOS ONE. 2017;12:e0169097.

35. Runtsch MC, O'Neill LAJ. GOTcha: IncRNA-ACOD1 targets metabolism during viral infection. Cell Res. 2018;28:137-138.

36. Yang $\mathrm{C}$, Wu K, Wang S, Wei G. Long non-coding RNA XIST promotes osteosarcoma progression by targeting YAP via miR-195-5p. J Cell Biochem. 2018;119(7):5646-5656.
37. Zhang YH, Fu J, Zhang ZJ, Ge CC, Yi Y. LncRNA-LINC00152 downregulated by miR-376c-3p restricts viability and promotes apoptosis of colorectal cancer cells. Am J Transl Res. 2016;8:5286-5297.

38. Zhao HX, Hou WG, Tao JG, et al. Upregulation of IncRNA HNF1A-AS1 promotes cell proliferation and metastasis in osteosarcoma through activation of the Wnt/beta-catenin signaling pathway. Am J Transl Res. 2016;8:3503-3512.

39. Zou ZW, Ma C, Medoro L, et al. LncRNA ANRIL is up-regulated in nasopharyngeal carcinoma and promotes the cancer progression via increasing proliferation, reprograming cell glucose metabolism and inducing side-population stem-like cancer cells. Oncotarget. 2016;7:61741-61754.

40. Su Y, Li L, Zhao S, Yue YN, Yang SX. The long noncoding RNA expression profiles of paroxysmal atrial fibrillation identified by microarray analysis. Gene. 2018;642:125-134.

41. Liu H, Dai CF, Wu QR, Liu HY, Li FT. Expression profiling of long noncoding RNA identifies Inc-MMP3-1 as a prognostic biomarker in external auditory canal squamous cell carcinoma. Cancer Med. 2017;6:2541-2551.

42. Zhang WY, Dong R, Diao S, Du J, Fan ZP, Wang F. Differential long noncoding RNA/mRNA expression profiling and functional network analysis during osteogenic differentiation of human bone marrow mesenchymal stem cells. Stem Cell Res Ther. 2017;8:30.

43. Wu CY, Liu H, Zhang FE, et al. Long noncoding RNA expression profile reveals IncRNAs signature associated with extracellular matrix degradation in kashin-beck disease. Sci Rep. 2017;7:17553.

44. Deng BY, Cheng X, Li HM, Qin JB, Tian ML, Jin GH. Microarray expression profiling in the denervated hippocampus identifies long noncoding RNAs functionally involved in neurogenesis. BMC Mol Biol. 2017;18:15.

45. Wan ZY, Song F, Sun Z, et al. Aberrantly expressed long noncoding RNAs in human intervertebral disc degeneration: a microarray related study. Arthritis Res Ther. 2014;16:465. https://doi. org/10.1186/S13075-014-0465-5

46. Lan PH, Liu ZH, Pei YJ, et al. Landscape of RNAs in human lumbar disc degeneration. Oncotarget. 2016;7:63166-63176.

47. Chen Y, Ni HJ, Zhao YC, et al. Potential role of IncRNAs in contributing to pathogenesis of intervertebral disc degeneration based on microarray data. Med Sci Monitor. 2015;21:3449-3458.

48. Zhao B, Lu M, Wang D, Li H, He X. Genome-wide identification of long noncoding RNAs in human intervertebral disc degeneration by RNA sequencing. Biomed Res Int. 2016;2016:3684875.

49. Wang XB, Lv GH, Li J, Wang B, Zhang QS, Lu C. LncRNA-RP11296A18.3/miR-138/HIF1A pathway regulates the proliferation ecm synthesis of human nucleus pulposus cells (HNPCs). J Cell Biochem. 2017;118:4862-4871.

50. Hiyama A, Sakai D, Risbud M, et al. Enhancement of intervertebral disc cell senescence by WNT/ $\beta$-catenin signaling-induced matrixmetalloproteinase expression.ArthritisRheum.2010;62:30363047.

51. Liang S, Zhang S, Wang P, et al. LncRNA, TUG1 regulates the oral squamous cell carcinoma progression possibly via interacting with Wnt/ $\beta$-catenin signaling. Gene. 2017;608:49-57.

52. Chen J, Jia YS, Liu GZ, et al. Role of LncRNA TUG1 in intervertebral disc degeneration and nucleus pulposus cells via regulating Wnt/beta-catenin signaling pathway. Biochem Biophys Res Comm. 2017;491:668-674.

53. Xi YH, Jiang TW, Wang WH, et al. Long non-coding HCG18 promotes intervertebral disc degeneration by sponging miR-146a-5p and regulating TRAF6 expression. Sci Rep. 2017;7(1):13234.

54. Zhang X, Hamblin M, Yin K. The long noncoding RNA Malat 1: its physiological and pathophysiological functions. RNA Biol. 2017;14:1705-1714.

55. Zhang H, Li JD, Duan DP, She W, Wang LG, Zhang FQ. The role of IncRNA MALAT1 in intervertebral degenerative disc disease. Int J Clin Exp Pathol. 2017;10:10611. 
56. Cui Y, Zhang F, Zhu C, Geng L, Tian T, Liu H. Upregulated IncRNA SNHG1 contributes to progression of non-small cell lung cancer through inhibition of miR-101-3p and activation of Wnt/ $\beta$-catenin signaling pathway. Oncotarget. 2017;8:17785-17794.

57. Tian T, Qiu R, Qiu X. SNHG1 promotes cell proliferation by acting as a sponge of miR-145 in colorectal cancer. Oncotarget. 2018;9:2128-2139.

58. Tan H, Zhao L, Song R, Liu Y, Wang L. The long noncoding RNA SNHG1 promotes nucleus pulposus cell proliferation through regulating miR-326/CCND1. Am J Physiol Cell Physiol. 2018; https://doi. org/10.1152/ajpcell.00220.2017 [Epub ahead of print].

59. Raveh E, Matouk I, Gilon M, Hochberg A. The H19 Long non-coding RNA in cancer initiation, progression and metastasis - a proposed unifying theory. Mol Cancer. 2015;14:184.

60. Wang X, Zou M, Li J, et al. LncRNA H19 targets miR-22 to modulate $\mathrm{HO}$-induced deregulation in nucleus pulposus cell senescence, proliferation, and ECM synthesis through Wnt signaling. J Cell Biochem. 2018;119(6):4990-5002.

61. Yu X, Li Z, Zheng H, Chan M, Wu W. NEAT1: a novel cancerrelated long non-coding RNA. Cell Prolif. 2017;50(2). https://doi. org/10.1111/cpr.12329.

62. Ruan Z, Ma H, Li J, Liu H, Jia H, Li F. The long non-coding RNA NEAT1 contributes to extracellular matrix degradation in degenerative human nucleus pulposus cells. Exp Biol Med (Maywood). 2018;243:595-600.

63. Sun Z, Yin Z, Liu C, Liang H, Jiang M, Tian J. IL-1 $\beta$ promotes ADAMTS enzyme-mediated aggrecan degradation through NF- $\mathrm{kB}$ in human intervertebral disc. J Orthop Surg Res. 2015;10:159.

64. Wang $\mathrm{K}$, Song $\mathrm{Y}$, Liu $\mathrm{W}$, et al. The noncoding RNA linc-ADAMTS5 cooperates with RREB1 to protect from intervertebral disc degeneration through inhibiting ADAMTS5 expression. Clin Sci. 2017;131:965-979.

65. Jathar S, Kumar V, Srivastava J, Tripathi V. Technological Developments in IncRNA Biology. In: Rao M, ed. Long Non Coding RNA Biology. Advances in Experimental Medicine and Biology. Vol 1008. Singapore: Springer; 2017;1008:283-323.

How to cite this article: Li Z, Li X, Chen C, et al. Long noncoding RNAs in nucleus pulposus cell function and intervertebral disc degeneration. Cell Prolif. 2018;51:e12483. https://doi.org/10.1111/cpr.12483 